\title{
String Physics and Black Holes
}

\author{
Leonard Susskind and John Uglum ${ }^{\mathrm{a}}$

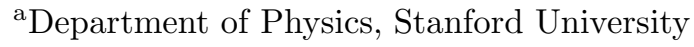 \\ Stanford, CA 94305-4060 USA
}

\begin{abstract}
In these lectures we review the quantum physics of large Schwarzschild black holes. Hawking's information paradox, the theory of the stretched horizon and the principle of black hole complementarity are covered. We then discuss how the ideas of black hole complementarity may be realized in string theory. Finally, arguments are given that the world may be a hologram.
\end{abstract}

\section{Introduction}

An outsider listening to this conference might get the idea that there is such a thing as string theory, that string theory is a relatively complete theory of the world, including gravitation. We do not believe this is so, at least not yet. There is a wide class of phenomena, perhaps the most interesting phenomena for future study, which string theory in its present formulation cannot address at this time, and perhaps cannot address at allPlanck scale physics.

The distinction between Planck scale physics and string scale physics is often ignored in string theory, but it is an imporant one. If $g$ is the string coupling and the number of large (uncompactified) spacetime dimensions is $D$, then the Planck scale $\ell_{P}$ is related to the string scale $\ell_{S}$ by the relation $\ell_{P}^{D-2}=g^{2} \ell_{S}^{D-2}$. It is usually assumed that the string coupling is very small, so there is a large difference between the two scales. Most of the phenomena that we are able to discuss in string theory-the spectrum, scattering amplitudes, etc. are all phenomena that have to do with the strings scale, not the Planck scale.

There is a host of problems at the Planck scale which the present formulation of string theory is simply incapable of handling. These include

- The thermodynamics of strings and their behavior at temperatures above the Hagedorn temperature [1]

- Very high energy scattering processes at very small impact parameter [2]
- Black hole evaporation and the puzzles associated with it

- The cosmological constant problem

In the case of a high energy central collision between two strings, it is easy to guess the answer-a black hole forms, and then evaporates. But we can't study this in string theory. If we did understand quantum gravity, then we could answer all of these questions.

The plan of these lectures is as follows. First, we will review the physics of horizons, including their thermal behavior, and all of the fundamental physics of large mass Schwarzschild black holes. We will see that all of the paradoxes associated with black hole evaporation can be addressed in this simple context, and attempts to resolve these paradoxes will lead us to the idea of black hole complementarity.

After reviewing the light front gauge formulation of strings, we will be able to ask how string theory might be able to resolve the paradoxes of black hole evaporation. In particular, we will be interested in how string theory stores information, and how the Planck scale is generated from string theory. We will discuss the entropy of horizons in string theory. Finally, we will discuss some ideas about the world as a hologram, due to 't Hooft and Susskind.

If one were to plot the distance scales that are probed as one increases the energy of a process, we know that for ordinary relativistic field theory, the length scale decreases as the energy increases. What we are now finding in string theory, how- 
ever, is that this behavior does not continue forever. Increasing the energy beyond the Planck energy, one starts to probe larger distances instead of smaller ones. This is the energy region which we must understand to solve the aforementioned problems.

\section{Schwarzschild Black Holes}

The line element for the eternal Schwarzschild black hole geometry, in Scwarzschild coordinates $(t, r, \theta, \varphi)$, is given by

$$
\begin{gathered}
d s^{2}=-\left(1-\frac{2 G M}{r}\right) d t^{2}+\left(1-\frac{2 G M}{r}\right)^{-1} d r^{2} \\
+r^{2} d \Omega^{2}
\end{gathered}
$$

where $G$ is Newton's constant, $M$ is the black hole mass, and $d \Omega^{2}$ is the line element of the unit twosphere. The surface $r=2 G M, t=\infty$ is the future event horizon. There is also a past event horizon at $r=2 G M, t=-\infty$, but we will not concern ourselves much with this, since for black holes formed by the gravitational collapse of matter it is absent. The singularity is at $r=0$.

Light signals from points outside the horizon can reach infinity, whereas light signals from points inside the horizon necessarily terminate when they reach the singularity. We can therefore think of the horizon as consisting of those photons which were just barely trapped by the black hole. Physics is complicated near the singularity, so we will restrict our attention to physics strictly outside the black hole. It is our belief that most of the interesting physics is at the horizon, anyway.

To a freely falling observer, there is nothing special about the horizon. All of the local geometrical invariants remain small at the horizon, so there is no local signal that he has crossed into the region of the black hole, and he can cross the horizon into the black hole in a finite amount of proper time. Note, however, that $t=\infty$ on the horizon, so an external observer, whose proper time is proportional to $t$, will never see anything cross the horizon. This is the first of a series of peculiar situations in which observers inside and outside will disagree.

Suppose we are interested in a region of space very close to the horizon, small compared to the size of the black hole, but large compared to any microscopic scales. (Equivalently, we might be interested in a black hole with very large $M$.) In this case, the Schwarzschild line element can be simplified. If we define $\rho$ to be the proper distance from the event horizon,

$$
\begin{aligned}
\rho= & \sqrt{r(r-2 G M)} \\
& +2 G M \ln \left(\frac{\sqrt{r-2 G M}+\sqrt{r}}{\sqrt{2 G M}}\right)
\end{aligned}
$$

and define a rescaled time coordinate $\omega=\frac{t}{4 G M}$, then for $r-2 G M \ll G M$, Eq. (1) can be approximated as

$d s^{2}=-\rho^{2} d \omega^{2}+d \rho^{2}+d y^{2}+d z^{2}$,

where $y$ and $z$ denote the directions tangent to the horizon. Eq. (3) is known as the Rindler metric, and is nothing more than flat Minkowski space in hyperbolic polar coordinates. If $(T, X, Y, Z)$ denote the coordinates of Minkowski space, then

$$
\begin{aligned}
T & =\rho \sinh (\omega), \\
X & =\rho \cosh (\omega), \\
Y & =y, \quad Z=z .
\end{aligned}
$$

A freely falling observer simply corresponds to an intertial Minkowski observer, and there is clearly nothing special about the horizon, which is just an ordinary light-like surface.

There is, however, something interesting about our parametrization of flat space. The time coordinate $\omega$ does not behave like an ordinary Minkowski time variable-in fact, it corresponds to a Lorentz boost parameter, and goes to infinity on the light like surface of the horizon. Spacelike surfaces of constant $\omega$ accumulate near the horizon. Observers at fixed $\rho$ describe hyperbolic trajectories, which means that they correspond to uniformly accelerated observers in Minkowski space. This makes sense, of course, since an observer who wants to remain outside the black hole must have a rocket or some other means of propulsion to keep from falling in. A diagram of Rindler space is shown in Fig. 1 .

The classical physics of observers who are restricted to remain only in the first quadrant of Rindler space, corresponding to the region outside the black hole, is completely consistent. Although signals which originate in quadrant IV can 


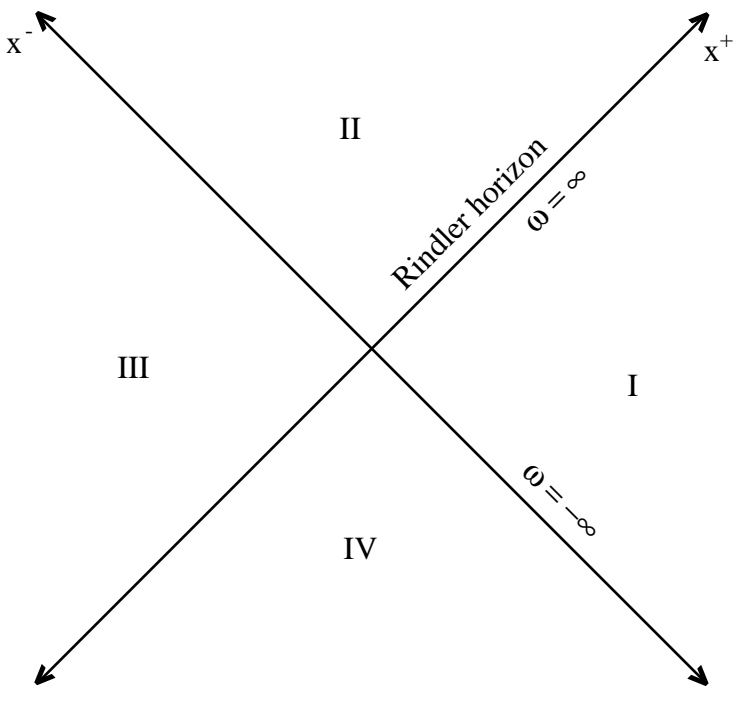

Figure 1. A diagram of Rindler space showing the four quadrants and the horizon.

influence events in quadrant $\mathrm{I}$, they must cross the past horizon at $\omega=-\infty$, and can therefore be treated as initial data. Similarly, signals which propagate out from quadrant I into quadrant II must cross the surface $\omega=\infty$. Quadrant III is simply out of causal contact with quadrant I, and signals originating there have no effect on events in quadrant I. When it comes to doing quantum mechanics in Rindler space, however, the story will get more complicated.

Now that we have some new intuition about the nature of spacetime near the horizon of a black hole, let's return to the full Schwarzschild metric and see if we can make some of the ideas obtained using the Rindler space approximation more precise. Let us first define the Regge-Wheeler tortoise coordinate

$r_{*}=r+2 G M \ln (r / 2 G M-1)$

and the Kruskal-Szekeres coordinates

$\begin{aligned} U & =-\exp \left(\left(r_{*}-t\right) / 4 G M\right), \\ V & =\exp \left(\left(r_{*}+t\right) / 4 G M\right) .\end{aligned}$

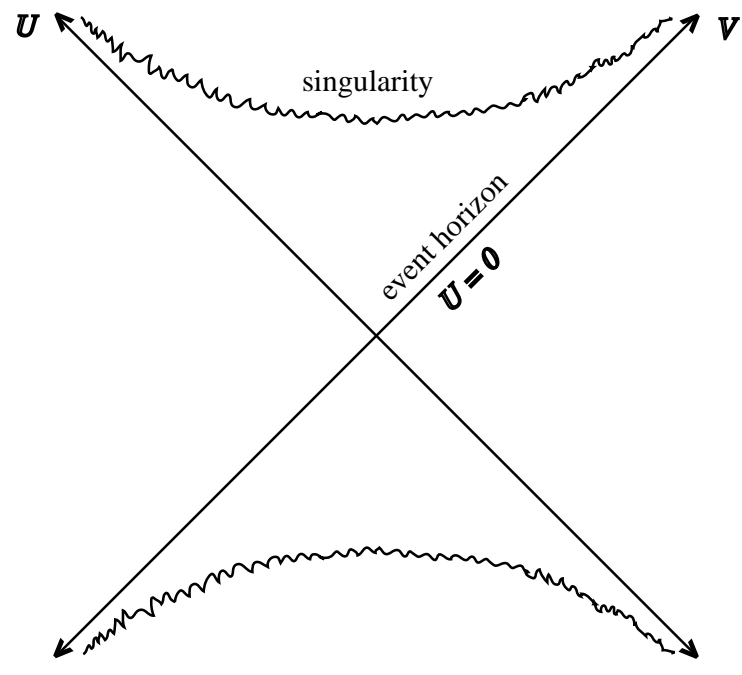

Figure 2. The Schwarzschild geometry in Kruskal coordinates.

Then the line element can be written

$d s^{2}=-\frac{32 G^{2} M^{3} e^{-r / 2 G M}}{r} d U d V+r^{2} d \Omega^{2}$.

The metric in the $(U, V)$ plane is conformal to flat space, and nothing special happens at the horizon $r=2 G M$. Note, however, that the singularity at $r=0$ has reappeared. Fig. 2 shows what the geometry looks like in the $(U, V)$ plane.

Using this diagram it is easy to understand the disagreement between freely falling and external observers. A freely falling observer simply follows a geodesic, crossing the horizon in a finite amount of proper time and eventually crashing into the singularity. When the freely falling observer crosses the horizon, he will no longer be able to send signals to the outside world. The external observer, in order to avoid the black hole, must be constantly accelerating, and therefore follows a hyperbolic trajectory. The horizon corresponds to a null surface which the external observer cannot intersect in a finite time, and thus must be at $t=\infty$. An external observer can only receive signals from points outside the hori- 
zon, and can thus never "see" anything cross the horizon.

In addition, as with the Rindler case, the surfaces of constant time accumulate near the horizon, and the time coordinate $t$ actually corresponds to a kind of Lorentz boost parameter. As $t$ increases, the relative boost between the external observer and an infalling particle increases. It is easy to show that as the particle falls toward the horizon, its momentum as seen by the external observer increases like $\exp (t / 4 G M)$. The black hole is the ultimate particle accelerator-the momentum of any particle as seen by the external observer will eventually become much larger than the Planck mass.

\section{Quantum Physics in Rindler Space}

Now let us consider the question of how to do quantum mechanics in Rindler space. Consider a single real free massless scalar field $\phi$ propagating in Rindler space. It will prove convenient to consider the wave equation for $\phi$ using the tortoise coordinates $\left(t, r_{*}, \theta, \varphi\right)$. The reason for this is that as $r_{*}$ runs from $-\infty$ to $\infty$, it covers the region from the horizon out to infinity. Thus it covers only the region outside the black hole. In these coordinates, the line element is

$d s^{2}=\left(1-\frac{2 G M}{r}\right)\left[-d t^{2}+d r_{*}^{2}\right]+r^{2} d \Omega^{2}$,

and we see that the $\left(t, r_{*}\right)$ part of the metric is conformal to flat space. Thus, for a massless scalar field, we expect the wave equation to take on a simple form. If we define $\psi=r \phi$ and expand $\psi$ in spherical harmonics $Y_{\ell}^{m}$, then the action for $\psi_{\ell}^{m}$ is

$$
\begin{gathered}
S=\sum_{\ell} \frac{1}{2} \int d r_{*} d t\left[\left(\partial_{t} \psi_{\ell}\right)^{2}-\left(\partial_{r_{*}} \psi_{\ell}\right)^{2}\right. \\
\left.-V_{\ell}\left(r_{*}\right)\left(\psi_{\ell}\right)^{2}\right]
\end{gathered}
$$

where

$V_{\ell}\left(r_{*}\right)=\frac{2 G M}{r}\left[\frac{\ell(\ell+1)}{r^{2}}-\frac{2 G M}{r^{3}}\right]$.

The new feature is $V_{\ell}$, which has the form of a position-dependent mass term for $\psi$. $V_{\ell}$ is the relativistic generalization of a centrifugal barrier, but it behaves differently than an ordinary centrifugal barrier, because while it is repulsive far from the black hole, it is attractive for $r<3 G M$. This means that particles of high angular momentum can be trapped in the region between the horizon and $r=3 G M$, and can rattle around in this region.

It is enlightening to examine the wave equation using the Rindler approximation. If we define a variable $u=\ln (\rho)$, then $u$ behaves like $r_{*}$, in that it goes to $\infty$ at asymptotic infinity and $-\infty$ at the horizon. We can Fourier expand the field $\phi$ to obtain transverse momentum modes $\phi_{\vec{k}}$. In the coordinates $(\omega, u)$, the Lagrangian for $\phi_{\vec{k}}$ takes a particularly simple form,

$\mathcal{L}=\frac{1}{2}\left[\left(\partial_{\omega} \phi_{\vec{k}}\right)^{2}-\left(\partial_{u} \phi_{\vec{k}}\right)^{2}-\vec{k}^{2} e^{2 u} \phi_{\vec{k}}^{2}\right]$.

The potential barrier is now given by $V_{\vec{k}}=\vec{k}^{2} e^{2 u}$, and we see that only the mode with $\vec{k}=0$ can escape to infinity.

The next important piece of information we will need is the thermal nature of Rindler space. Specifically, we will see that a Rindler observer describes the ordinary Minkowski vacuum by a thermal density operator. This is a general result, not restricted to the above case of a massless scalar field. Consider dividing the hypersurface $T=0$ of Minkowski space into two halves, one with $X<0$, and one with $X>0$. We will call these halves the left and right halves, respectively. Assume that the Hilbert space $\mathcal{H}$ on the hypersurface $T=0$ factorizes into a product space $\mathcal{H}_{L} \otimes \mathcal{H}_{R}$. If $\left\{|b\rangle_{L}\right\}$ is an orthonormal basis for $\mathcal{H}_{L}$ and $\left\{|a\rangle_{R}\right\}$ is an orthonormal basis for $\mathcal{H}_{R}$, then a general ket $|\psi\rangle$ in $\mathcal{H}$ can be written

$|\psi\rangle=\sum_{b, a} \psi(b, a)|b\rangle_{L} \otimes|a\rangle_{R}$.

If we now trace over the degrees of freedom in $\mathcal{H}_{L}$, the resulting density matrix for the right half of the hypersurface is given by

$\rho\left(a, a^{\prime}\right)=\sum_{b} \psi(b, a) \psi^{*}\left(b, a^{\prime}\right)$.

Since we argued that no causal signal from the hypersurface $T=0, X<0$ can enter quadrant 
I, the complete set of states on the hypersurface $T=0, X>0$ is in fact the complete set of states needed to describe physics in Rindler space for all time. Thus, the above construction is precisely the density matrix used by a Rindler observer in quadrant I.

Now consider performing this decomposition for the Minkowski ground state $|0\rangle$. Given some arbitrary set of fields, which we will denote by $\phi$, we can represent the ground state wave functional $\Psi_{0}(\phi)$ by using the Feynman-Kac formula,

$\Psi_{0}(\phi)=\int_{\mathcal{F}}[d A] e^{-I[A]}$,

where $I$ is the Euclidean action for the field and the integral is over the set $\mathcal{F}$ of functions defined for $T \geq 0$ and which match $\phi$ at $T=0$. Let $H_{R}$ denote the Rindler space Hamiltonian for the field $\phi$, which generates translations in $\omega$. Since $\omega$ corresponds to hyperbolic boost angle in the $(T, X)$ plane of Minkowski space, when we Wick rotate to Euclidean space, $H_{R}$ becomes the generator of rotations in the $(T, X)$ plane. Thus we can write

$\Psi_{0}(\phi)={ }_{L}\left\langle\phi_{L}\left|\exp \left(-H_{R} \pi\right)\right| \phi_{R}\right\rangle_{R}$.

Thus the density matrix for the Minkowski vacuum, $\rho_{0}\left(\phi_{R}, \phi_{R}^{\prime}\right)$, is given by

$$
\begin{aligned}
\rho_{0} & =\sum_{\phi_{L}}{ }_{R}\left\langle\phi_{R}\left|e^{-\pi H_{R}}\right| \phi_{L}\right\rangle_{L L}\left\langle\phi_{L}\left|e^{-\pi H_{R}}\right| \phi_{R}^{\prime}\right\rangle_{R} \\
& ={ }_{R}\left\langle\phi_{R}\left|e^{-2 \pi H_{R}}\right| \phi_{R}^{\prime}\right\rangle_{R},
\end{aligned}
$$

and so the density operator for the Minkowski space vacuum is $\rho_{0}=\exp \left(-2 \pi H_{R}\right)$, which is indeed thermal, with inverse temperature $\beta=2 \pi$.

This phenomenon is known as the Unruh effect: accelerating observers experience thermal radiation. But is there any sense in which the Rindler observer is actually experiencing a bath of thermalized particles? Would a real thermometer measure a temperature? The answer is yes. Consider the fact that there are always fluctuations of the vacuum. These fluctuations can be described as loops in spacetime. Some of these loops will encircle the origin, lying partially inside and partially outside quadrant I. But since these loops intersect the surfaces $\omega=-\infty$ and $\omega=\infty$, as far as the Rindler observer is concerned, they are particles which are present for all time. The Rindler observer sees these fluctuations as a bath of thermal particles which are ejected from the horizon infinitely far in the past and which will eventually fall back onto the horizon in the infinite future.

Note however that this is an interpretation of a particular phenomenon by a particular observer. A freely falling inertial observer would not describe these vacuum fluctuations in the same way. In fact, the freely falling observer would not be able to distinguish these fluctuations from any other vacuum fluctuations, and would notice nothing out of the ordinary. It is only the Rindler observer who can distinguish this thermal radiation, and only the Rindler observer who must describe the vacuum using a thermal density matrix.

The proper temperature measured by a Rindler observer at distance $\rho$ from the horizon can be obtained from the Rindler temperature $T_{R}=1 / 2 \pi$ by using the transformation between Rindler time and proper time. The proper temperature is thus given by $T_{\text {proper }}=1 / 2 \pi \rho$. A Rindler observer will therefore describe the region close to the horizon as a very hot place, and in order to describe physics in this region, he will have to understand physics at extremely high temperatures. If the Rindler observer uses an effective theory with some cutoff mass $\Lambda$, then it is natural to impose a cutoff at a distance of order $1 / \Lambda$ from the horizon, beyond which the observer cannot penetrate. For consistency, however, this boundary surface must be endowed with some set of degrees of freedom which represent the degrees of freedom integrated out to obtain the effective theory at scale $\Lambda$, and should behave like a hot membrane. This surface effectively augments the horizon, and is known as a stretched horizon. We will return later to this very important idea.

Now that we have determined that a Rindler observer experiences a temperature which decreases as one moves away from the horizon, let us return to the quantum fields. For each transverse momentum mode $\vec{k} \neq 0$, the field $\phi_{\vec{k}}$ is excited to a thermal spectrum. Each mode is populated according to the Boltzmann distribution with tem- 
perature $1 / 2 \pi$, so the modes with energies greater than $1 / 2 \pi$ will be exponentially suppressed. But for energies less than $1 / 2 \pi$, there will exist a bath of thermal particles which create a thermal atmosphere around the black hole. Only for the mode with $\vec{k}=0$ can these particles escape to infinity.

As we saw earlier, Rindler space is a good description of any region of the horizon small compared to the entire black hole. Let us then consider the interpretation of these results for the finite mass black hole. High angular momentum particles which are ejected from the region close to the horizon are deflected by the centrifugal barrier and become part of the thermal atmosphere of the black hole. Only the lowest angular momentum modes can escape the centrifugal barrier. This slow leakage of particles out of the centrifugal barrier is known as Hawking radiation [3], and leads to the eventual evaporation of the black hole. The temperature as observed by an observer at asymptotic infinity is $T_{\infty}=T_{R} d \omega / d t=1 / 8 \pi G M$, which is known as the Hawking temperature. This implies an evaporation time for the black hole of order $G^{2} M^{3}$. The fact that only the lowest angular momentum modes can escape is the reason for the long evaporation time of the black hole.

\section{Gedanken Experiments Involving Black Holes}

In the previous sections, we have argued that an external observer can describe the black hole as a hot membrane which can absorb and emit particles. Let us now consider a gedanken experiment designed to test the existence of the stretched horizon [ [.

Suppose that physics below some energy scale $\Lambda$ can be described by a more-or-less standard grand unified theory. If the claim that the stretched horizon behaves like a hot membrane is correct, then an observer near enough to the horizon ought to be able to detect baryon number violation. Now, we can make the black hole as large as we like-galactic size, for example-so all the tidal forces at the horizon are exceedingly small, and it is hard to imagine how we are going to see any baryon number violation. Neverthe- less, let us press on and see what we find.

Imagine constructing what we call a "GUT bucket". The GUT bucket is sealed, so that no GUT particles can enter or leave the container, and has the property that it can withstand GUT scale temperatures, but not Planck scale temperatures. Although we know of no way to construct such a GUT bucket, there is no reason to think that such a container in any way violates the laws of physics, so it should be perfectly fine to perform gedanken experiments with it. We can imagine that the bucket is suspended above the black hole by some mechanism like a winch, and that we can slowly lower the bucket toward the surface of the black hole.

The experiment we will perform, then, is to start off with the bucket far away from the horizon, where temperatures are far less than the GUT scale, with no baryons in it. We will then slowly lower the bucket down toward the horizon until it has reached a distance of order $1 / \Lambda$, and after some time, slowly raise the bucket back to the region far from the horizon. We will then open the bucket and find out if baryon number has been violated.

If we do this many times, on the average we will find that baryon number has not been violated, but for any given individual trial, we can expect to see baryon number violation. The theory behind this is quite simple. In order to suspend the GUT bucket a distance $\Lambda$ from the horizon, we must subject the GUT bucket to an acceleration of order $1 / \Lambda$ to prevent it from falling into the black hole. This acceleration disturbs the vacuum within the bucket on frequency scales of order $\Lambda$, and the interactions of these vacuum fluctuations with the walls of the bucket, etc. can produce baryon number violating effects.

Suppose we now change the experiment a little: suppose we allow the bucket to fall through the horizon freely. As we stated earlier, there are no large gravitational or tidal forces at the horizon of a big black hole, so there is no local signal that the bucket has fallen through the horizon, and an observer in the bucket will not be able to detect any baryon number violation. On the other hand, once the bucket has fallen through the horizon, it cannot report to the outside world that 
there was no baryon number violation, so there is no contradiction here. An outside observer cannot derive a contradiction because he cannot get the information that there was no baryon number violation, and the observer inside the bucket detects no baryon number violation until he meets his demise at the singularity.

So, can we change the experiment to try and obtain a contradiction? Suppose we prepare another GUT bucket, which we will allow to fall freely through the horizon, but this time we program the bucket to send out a message when it has reached a distance $1 / \Lambda$ from the horizon to tell us whether or not there is any baryon number violation. If, however, the experimental apparatus and signalling device all operate by sending out electromagnetic radiation (i.e. they work as fast as they possibly can), then the entire process of measurement and signalling must occur within a time interval $1 / \Lambda$. This process introduces frequencies of order $\Lambda$ into the measurement and signalling processes, so the very act of measuring and reporting on the baryon number within the bucket in a time interval of order $1 / \Lambda$ can, in fact, change the baryon number within the bucket. This is a classic example of quantum mechanical complementarity.

There is a second and perhaps more convincing way to understand the results of the above experiment. Let us for the moment igore questions of confinement, and suppose that we are able to put a single quark into the GUT bucket. Let us suppose further that the GUT theory has dimensionless coupling $\alpha$ at the scale $\Lambda$, which governs the strength of an interaction between quarks, antiquarks, and X-bosons. This is a baryon number violating interaction. In what sense, then, is baryon number conserved? If we were to calculate the fraction of a given interval of time that a quark spends as an anti-quark, that is, the fraction of the time spent "in the wrong state", we would find that this fraction is non-negligible, and is in fact proportional to $\alpha$. The point is, however, that these virtual transitions occur very fast-on time scales of order $1 / \Lambda$. If we define a time averaged baryon number, where we average over intervals large compared to $1 / \Lambda$, then we would find that it is this averaged or effective baryon number which is very hard to change. It is in this sense that the baryon number violating interactions are unimportant for low energy physics, because we need high energy interactions to notice them.

Now suppose that the quark in our GUT bucket makes such a transition as it is passing through the horizon. This will occur in roughly a fraction $\alpha$ of the trials. Thus, during the entire time at which the measurement is occuring, the quark is in the wrong state, and the measurement apparatus will report this result. We see, therefore, that it is the uncertainty principle of quantum mechanics, and nothing to do with gravitational forces, that causes the experiment to report baryon violation.

A third way of understanding this is to draw the Feynman diagram of a quark undergoing this transition. If the loop representing the virtual states does not lie entirely within quadrant I of Rindler space, then the virtual states necessarily intersect either $\omega=-\infty$ or $\omega=\infty$. Thus, from point of view of a Rindler observer, the transition is not virtual at all, but represents an interaction which changes a quark into an anti-quark and Xboson, which remain in the Rindler spacetime for all time.

There are other gedanken experiments one can devise to test the existence of the stretched horizon from the external observer's point of view 何. In general, one finds that the description is consistent with the known laws of physics, and any apparent paradoxes can be traced to unwarranted assumptions about the nature of physics at the Planck scale.

\section{The Stretched Horizon and Black Hole Complementarity}

Throughout the last few sections, we have developed some new insight into the way a black hole can be described by an external observer. This description is in terms of a stretched horizon, which we understand loosely as a very hot membrane just above the black hole surface. From the point of view of an external observer, the stretched horizon can interact with infalling matter, and can absorb and thermalize information possessed by that matter. Let us now summarize 
what is known about the stretched horizon. Much of this was developed in the book by Thorne, Price, and MacDonald [5].

The stretched horizon can be summarized as a membrane which lies just above the event horizon. In the formulation of [5], the exact distance of this membrane above the horizon is somewhat arbitrary, and is generally fixed for convenience. For example, if the outside observer performs measurements which are sensitive to energies up to the weak scale, then the stretched horizon may profitably be thought of as lying at a distance of about the weak scale from the event horizon. The interactions of the stretched horizon with the outside world can be thought of as arising from the boundary conditions that must be implemented in the cutoff theory. More recently, studies of black hole horizons in the context of string theory have shown that the stretched horizon is most naturally thought of as lying at the string scale above the event horizon, where the local temperature becomes of order the Hagedorn temperature, as seen below.

The temperature of the horizon is given by $T_{S H}=1 / 2 \pi \rho_{S H}$, where $\rho_{S H}$ is the proper distance from the event horizon to the stretched horizon. This is a universal temperature for all non-extremal black holes for a given $\rho_{S H}$. Remember that we found that the centrifugal barrier causes almost all of the particles emitted from the black hole to be deflected back in, so these particles form a thermal bath above the black hole horizon. The fact that very few of the particles get out means that the black hole evaporation process is very slow, so the idea of thermal equilibrium and temperature is well defined.

The horizon has other interesting physical properties as seen by an external observer. It has an electrical resistivity. If you place two electical leads on the stretched horizon and measure the resistance, you find a resistivity of 377 ohms/square.

The horizon has an entropy per unit area, given by the Bekenstein-Hawking formula [6,7]

$\frac{S}{A}=\frac{1}{4 G}$.

This formula is easily obtained from the thermo- dynamic relation $d E=T d S$, where the energy $E$ of the black hole is simply its mass $M$, the temperature is given by $T=1 / 8 \pi G M$, and the area of the horizon is $A=16 \pi G^{2} M^{2}$. (There is a constant of integration which comes from this formula, which gives a subleading term which will not concern us much here. We are not going to entertain the possibility that this constant is infinite.)

The horizon also has an energy per unit area,

$\frac{E}{A}=\frac{1}{8 \pi G \rho_{S H}}$,

which is obtained as follows. The Poisson bracket between Schwarzschild time and energy is given by $\{t, M\}=1$. Now consider an observer at rest very close to the horizon. The proper time of an observer at proper distance $\rho$ from the horizon is given to leading order in $\rho$ by

$\tau=\frac{t}{4 G M \rho}$.

Let the energy of the black hole as measured by this observer be $E$. Then we have the Poisson bracket relation $\{\omega, E\}=1$. Writing $E(M)$, we obtain the relation

$\{t, E(M)\}=4 G M \rho$.

Integrating up this equation gives

$E(M)=2 G M^{2} \rho$,

and thus $E / A=1 / 8 \pi G \rho$. Setting $\rho=\rho_{S H}$ gives the result Eq. $(18)$.

The horizon also has both bulk and shear viscosity. If an object falls into black hole, it will deform the surface of the black hole, and the deformation will propagate around the surface as would a disturbance in a viscous liquid. So, it is starting to sound like the properties of the horizons of all non-extremal black holes are universal, and indeed this is so. There is something strange about all this, though, in view of the fact that a freely falling observer will not see any of this. The conclusion is that there is no invariance to the existence of the stretched horizon-its existence depends upon one's state of motion. 
We would now like to introduce a principle, called the Principle of Black Hole Complementarity. This is a speculative principle which was introduced to solve the black hole information problem [8]. The principle can be stated as follows.

- From the point of view of an external observer, the stretched horizon exists and is a collection of quantum mechanical, microscopic degrees of freedom which can absorb, store, thermalize, and emit any quantum mechanical information which falls into the black hole.

- A freely falling observer will not detect the stretched horizon, nor will he experience any other local signal when he crosses the horizon.

We saw above in Chapter 4 that there are no obvious internal inconsistencies in the principle of black hole complementarity, although there are some serious arguments why it is wrong. but before we examine these arguments, let us digress for a moment and examine the connection between information, entropy, and how normal thermodynamic systems are supposed to behave.

\section{Information and Entropy}

We have not really defined information yet, although we have alluded to the paradox of information loss in black hole evaporation. Let us first consider the concept of entropy of entanglement between two quantum systems. Suppose we have two quantum systems, $A$ and $B$, and suppose the combined system is in the state $|\psi\rangle \in \mathcal{H}=\mathcal{H}_{A} \otimes \mathcal{H}_{B}$. As we saw in section 3, if we trace over the degrees of freedom in $\mathcal{H}_{B}$, we obtain a density operator $\rho$ whose matrix elements are given by

$\rho_{A}\left(a, a^{\prime}\right)=\sum_{b} \psi(a, b) \psi^{*}\left(a^{\prime}, b\right)$.

Given such a density matrix, one can obtain the entropy of entanglement between $A$ and $B$, which is defined to be

$S_{A}=-\operatorname{tr}\left(\rho_{A} \ln \left(\rho_{A}\right)\right)$.
It is easy to see that $S_{A}=S_{B}$. The entropy of entanglement is essentially the logarithm of the number of independent states which have a nontrivial probability of being occupied. This entropy arises because the systems have non-trivial correlations with each other.

There is another kind of entropy, which we will call entropy of ignorance. This entropy is not there because the system is necessarily entangled, but simply because you have not measured all the possible variables needed to completely specify the state of the system. For example, thermodynamic entropy is of this type. For a thermal system, instead of using a density operator obtained from tracing out microscopic degrees of freedom, one simply postulates a density operator of the form $\rho=\exp (-\beta H) / Z(\beta)$, where $Z(\beta)$ is the partition function. In general, $S_{\text {entanglement }} \leq S_{\text {thermal }}$, because $S_{\text {thermal }}$ represents not only the entanglement of the system with its environment, but also entropy our ignorance of it. The information $I$ contained in a system can be defined as the difference between the maximum entropy of ignorance, usually taken to be the thermodynamic entropy, and the entanglement entropy, so $I=S_{\max }-S_{E}$.

To understand how entropy and information behave in ordinary systems, let us now consider a gedanken experiment due to Sidney Coleman. Consider a lump of black coal, which we will treat as an ideal black body, at zero temperature. Let us illuminate the coal with a sequence of pulses from a laser beam. This heats up the coal, which begins to glow and radiate away the energy absorbed from the laser in the form of thermal radiation. This continues until the coal has cooled back down to its ground state. Since we know that the $S$-matrix for this process exists and is unitary, it must be true that the information coded in the sequence of pulses must still be present. Since the coal has cooled back down to zero temperature, it contains no information, so the information must be contained in the radiation field. So we see that thermal radiation can, in fact, code information, although we have not determined the mechanism by which it does this.

This question was analyzed in a brilliant paper by Don Page [9], whose results imply that the 


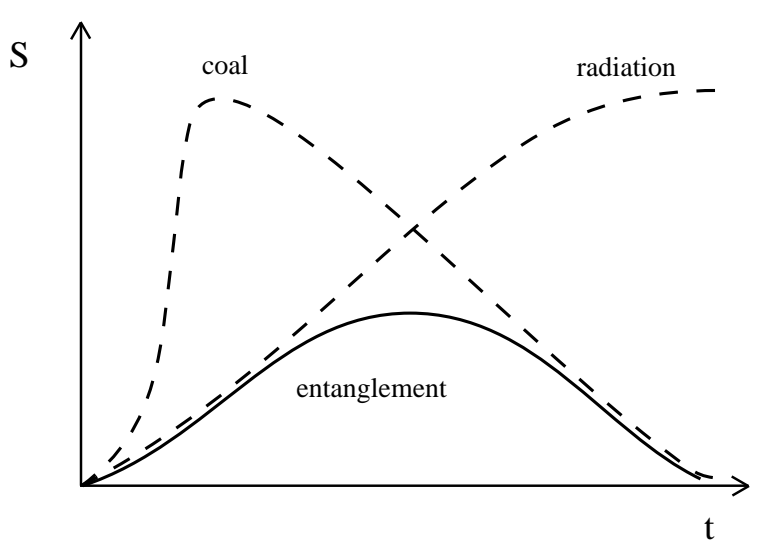

Figure 3. Plot of thermal and entanglement entropies of two subsystems.

information is in fact coded in long-time, longdistance correlations between the photons. The analysis went something like this. Consider the combined quantum system composed of the radiation field and the lump of coal. Initially, the thermal entropy of the coal is very large, while that of the radiation field is very small. In Fig. 3 we have plotted the thermal and entanglement entropies of the systems as functions of time. The entanglement entropy, common to both systems, must be less than either of their thermal entropies. Page found that the entanglement entropy is almost exactly equal to the thermal entropy of the radiation field, up to the point where the thermal entropies of the radiation and the coal are equal. At the crossover point, the entanglement entropy comes within about one bit of the thermal entropy, and then begins decreasing. It closely follows the thermal entropy of the coal down to zero. At this point, the systems are completely unentangled, but there is a large amount of thermal entropy (i.e. entropy of ignorance) in the radiation field. The information represented by this entropy contains the information coded in the sequence of laser pulses.

The question Page asked was, how much of the combined system must we measure before we can get any information out? The answer is that we must be able to sample at least half of the combined system before we can obtain even one bit of information. This shows that the information is not contained in short distance correlations, which could be distinguished by examining small portions of the system, and so must be contained in long-distance correlations. From this, we see that the information in the laser beam is first stored by the coal, and then transferred to the radiation field in the form of long-distance (or long-time) correlations between the photons.

Of course, we are interested in black holes, not coal. The point is that the principle of black hole complementarity implies that black holes behave exactly the same way. The information which is contained in the infalling matter is thermalized by the stretched horizon, and re-emitted in the Hawking radiation. But to decode the initial information, one must look at very long time correlations between the Hawking photons. To retrieve even one bit of the initial information, even in principle, one must wait for a time of order half the lifetime of the black hole. In reality, decoding the information from the radiation field is an extraordinarily complicated process (as in the case of the coal).

\section{Formal Arguments Against Black Hole Complementarity}

The first argument we shall consider concerns the location of information in the plane of the horizon. Suppose we have a large black hole, and suppose we allow a neutron to fall through the horizon at transverse location $\left(\theta_{0}, \varphi_{0}\right)$. Standard calculations in quantum field theory show that, after the neutron has fallen through the stretched horizon, the information needed to distinguish the particle as a neutron (i.e. $S U(3) \times S U(2) \times$ $U(1)$ quantum numbers, baryon number, lepton number, etc.) can be obtained by measuring a small region around $\left(\theta_{0}, \varphi_{0}\right)$. This certainly contradicts the idea that the stretched horizon contains microscopic degrees of freedom which thermalize and scramble all the infalling information.

If black hole complementarity is to be correct, then it must be the case that some new 
physics enters which thermalizes the information contained by the neutron, and causes it to spread out over the horizon. This is a necessary condition for a theory which implements black hole complementarity. We will return to this condition in the context of string theory shortly.

A second argument concerns the longitudinal behavior of matter as it falls toward the horizon. In Chapter 5 we argued that the horizon has an entropy per unit area given by Eq. (17), and in Chapter 6 we argued that the entropy should be interpreted as the logarithm of the number of accessible microstates of the system. Now consider sending a particle toward a black hole. In the frame of an external observer, the momentum of the particle increases exponentially with time. According to the usual Lorentz contraction formula, the fields associated with the particle will contract in the longitudinal direction (direction of motion), and this contraction will proceed indefinitely, since the relative boost increases without bound. Thus the longitudinal region occupied or influenced by the particle can be made arbitrarily small.

In the frame of the external observer, no particle ever crosses the horizon. Since we have argued that the longitudinal extent of particles can be made arbitrarily small, we see that, in effect, we can stack an arbitrarily large number of particles in layers of ever decreasing thickness onto the horizon. This means that the number of accessible states per unit area, and thus the entropy per unit area, can be made arbitrarily large, in contrast to the finiteness of the entropy per unit area given by Eq. (17). Thus, if the entropy per unit area is to have any type of state counting interpretation, there must be a mechanism which halts the Lorentz contraction as some point, and prohibits us from stacking an arbitrarily large amount of information into the horizon. This provides another necessary condition for a theory to implement black hole complementarity.

We will now give an argument for information loss based on effective low energy field theory, called the nice slice argument. The precise formulation of the argument which we will use is due to Joe Polchinski, but the argument is implicit in the literature dating back many years.
Consider the geometry for the formation of a large black hole by infalling matter, and imagine foliating the geometry by a family of Cauchy surfaces that have the following properties. First, the momenta of all the infalling particles are small in the frame of the surfaces. Second, the outgoing Hawking radiation has small momentum in the frame of the surfaces, up until the point that the black hole has become so small that the semiclassical approximation breaks down. Finally, the surfaces are everywhere smooth, so that there are no large local geometric quantities. We will call such a family of Cauchy surfaces a family of nice slices. The first construction of a family of nice slices that we are aware of is due to Wald.

It is now easy to give the argument for information loss. Since all momenta relevant to the black hole formation and evaporation processes are small in the nice slice frame, and the adiabatic theorem guarantees that all the high energy modes will be in their ground states, we can use low energy effective field theory to describe the process. Consider some information encoded in local correlations of the effective fields which propagates into the black hole. The equivalence principle states that nothing out of the ordinary happens to the infalling wave packet as it crosses the horizon, so a local observer falling with it must necessarily be able to retrieve the information after he has entered the black hole. This shows that the information is certainly inside the black hole.

We now invoke the so-called "no quantum Xerox principle". Suppose that the Hilbert space of states $\mathcal{H}$ factorizes into $\mathcal{H}_{\text {in }} \otimes \mathcal{H}_{\text {out }}$, and suppose that we try to build a quantum Xerox machine that replicates the information contained in a state $|\psi\rangle_{\text {in }} \in \mathcal{H}_{\text {in }}$ into a state in $\mathcal{H}_{\text {out }}$. The quantum Xerox machine must be a linear operator on the Hilbert space, which we will denote $X$. Then our rule is

$X\left[|\psi\rangle_{\text {in }} \otimes|\gamma\rangle_{\text {out }}\right]=|\psi\rangle_{\text {in }} \otimes|\psi\rangle_{\text {out }}$

for any state $|\gamma\rangle_{\text {out }} \in \mathcal{H}_{\text {out }}$. Now we can always write $|\psi\rangle_{i n}=|\alpha\rangle_{i n}+|\beta\rangle_{i n}$ for some $|\alpha\rangle_{i n}$ and $|\beta\rangle_{\text {in }} \in \mathcal{H}_{\text {in }}$. But then

$$
X\left[|\psi\rangle_{\text {in }} \otimes|\gamma\rangle_{\text {out }}\right]
$$




$$
\begin{aligned}
& =X\left[\left(|\alpha\rangle_{\text {in }}+|\beta\rangle_{\text {in }}\right) \otimes|\gamma\rangle_{\text {out }}\right] \\
& =X\left[|\alpha\rangle_{\text {in }} \otimes|\gamma\rangle_{\text {out }}\right]+X\left[|\beta\rangle_{\text {in }} \otimes|\gamma\rangle_{\text {out }}\right] \\
& =|\alpha\rangle_{\text {in }} \otimes|\alpha\rangle_{\text {out }}+|\beta\rangle_{\text {in }} \otimes|\beta\rangle_{\text {out }} \\
& \neq|\psi\rangle_{\text {in }} \otimes|\psi\rangle_{\text {out }} \mid>.
\end{aligned}
$$

Thus we see that the operator $X$ is not in fact a linear operator, and so is not admissible. This shows that there is no such thing as a quantum Xerox machine.

Now let us show why information must be lost. Since we are able to describe the black hole evaporation process using effective local fields which commute at spacelike separation, the Hilbert space of the effective field theory factorizes into the product $\mathcal{H}_{\text {in }} \otimes \mathcal{H}_{\text {out }}$. The no quantum Xerox machine principle then applies, stating that if the information contained by infalling matter is certainly contained within the black hole, then it is certainly not contained in the region outside the black hole. Moreover, since the fields commute at spacelike separation, there is no way for the information to get outside the black hole once it has entered. Thus, the information cannot escape and is lost to the external observer.

This seems to be an air-tight argument for information loss, but it includes a crucial assumption, which is that the low energy theory is in fact a local field theory. That this is the case is not at all obvious, especially in string theory, so there is still hope. In the next section we begin our examination of string theory, where we will see that there are indications that black hole complementarity is realized in string theory.

\section{Light-Front String Theory and Comple- mentarity}

String theory is a theory of strings, not particles, and it behaves very differently from ordinary quantum field theories in many respects. In this section we will analyze the physics of stringy matter falling toward a horizon as described by an external observer, and will see that there is circumstantial evidence that black hole complementarity is realized in string theory. Specifically, we will show that the necessary conditions of transverse spreading and the cessation of longitudinal Lorentz contraction both occur in string theory.
Although we presently do not have the technology to quantize string theory in a black hole background, we saw earlier that in the vicinity of the horizon, the black hole geometry is well approximated by Rindler space, so we shall use that approximation. Then we simply have strings in flat space, which we understand how to deal with.

We will use the light-front gauge formalism of string theory. There are a number of reasons why the light-front formalism is convenient for this problem. Imagine a single string falling through a Rindler horizon. Since the surfaces of constant Rindler time $\omega$ are obtained by boosting the surface $T=0$, it is clear that, given any inertial frame, the surfaces of constant $\omega$ become almost light-like with respect to this frame for $\omega$ large enough. Thus, evolution in Rindler time for large $\omega$ is well approximated by evolution in light-front time.

An important fact to notice is that the relation between proper time $\tau$ in the frame of the string and Rindler time $\omega$ is given for $\omega$ large by

$\frac{d \tau}{d \omega}=\rho e^{-\omega}$.

Therefore, as $\omega$ gets large, less and less proper time in the frame of the string elapses per unit of Rindler time, so a Rindler observer "sees" the string with an ever increasing time resolution. More precisely, if a Rindler observer at fixed proper distance from the horizon samples outgoing photons at a uniform rate of one sample per unit Rindler time, then the world lines of the sampled photons form a sequence of light like surfaces which accumulate at the horizon at the rate given by Eq. (26).

There is another way to think of this. We have already seen that the momentum of an infalling string, as reckoned by an external observer, increases like $e^{\omega}$ as $\omega$ gets large. The increased time resolution can simply be thought of as the Lorentz contraction of time between the two frames.

Let us now review some of the fundamentals of light-front gauge string theory. We will work in units where $\alpha^{\prime}=1 / 2$. The world sheet coordinates are taken to be $(\tau, \sigma)$, where $\tau$ is the timelike direction, and the metric on the world sheet is just the usual two-dimensional Minkowski metric. 
We define light front coordinates in spacetime by

$X^{ \pm}=\frac{1}{\sqrt{2}}\left(X^{0} \mp X^{D-1}\right)$,

and choose the gauge $X^{+}=\tau$. 1 After gauge fixing, the longitudinal coordinate $X^{-}$is completely determined in terms of the transverse coordinates $\vec{X}$, and the action for the transverse coordinates is simply

$S=\frac{1}{2 \pi} \int d \tau d \sigma\left[\left(\partial_{\tau} \vec{X}\right)^{2}-\left(\partial_{\sigma} \vec{X}\right)^{2}\right]$.

In addition, we have the condition that $p^{+}$, the longitudinal momentum, is conserved, and is uniformly distributed along the string. For convenience, we will set $p^{+}=1$. The transverse string coordinates can be expanded as

$\vec{X}(\sigma)=\vec{x}+\frac{i}{2} \sum_{n \neq 0} \frac{1}{n}\left[\vec{\alpha}_{n} e^{2 i n \sigma}+\tilde{\hat{\alpha}_{n}} e^{-2 i n \sigma}\right]$

where $\vec{x}$ is the center of mass position of the string at $\tau=0$ and the $\alpha_{m}^{i}$ obey the commutation relations

$\left[\alpha_{m}^{i}, \alpha_{n}^{j}\right]=m \delta^{i j} \delta_{m+n}$.

Let us now use light-front string theory to answer the question, how big is a string? Specifically, we will calculate the mean square transverse size of a closed bosonic string in its ground state. This is given by the matrix element

$\left\langle 0\left|(\vec{X}(0)-\vec{x})^{2}\right| 0\right\rangle$.

Explicit calculation of Eq. (31) using the mode expansion Eq. (29) yields

$\left\langle 0\left|(\vec{X}(0)-\vec{x})^{2}\right| 0\right\rangle=\frac{D-2}{2} \sum_{n=1}^{\infty} \frac{1}{n}$

which diverges logarithmically. Thus our calculation leads to the idea that the string has an infinite transverse extent. But what does this mean? Is this another infinity which should be renormalized somehow? The answer is no - let us

\footnotetext{
${ }^{1}$ Note that the usual convention is $X^{ \pm}=\frac{1}{\sqrt{2}}\left(X^{0} \pm\right.$ $X^{D-1}$ ), but the above choice is more convenient for our purposes.
}

see why. Consider an actual experiment designed to measure the transverse size of a string. Such an experiment will take some amount of time $\varepsilon$ to perform, and thus will be insensitive to frequencies greater than $N \sim 1 / \varepsilon$. Thus the mode sum in Eq. (32) should be cut off at $N$, giving a finite but $\varepsilon$-dependent answer,

$\left\langle 0\left|(\vec{X}(0)-\vec{x})^{2}\right| 0\right\rangle=\frac{D-2}{2} \sum_{n=1}^{N} \frac{1}{n}$,

which behaves like $\log (1 / \varepsilon)$. We see that as the resolution time $\varepsilon$ goes to zero, the measured size of the string diverges logarithmically. This behavior is nothing more than the Regge nature of string scattering amplitudes. It is also possible to show that the total measured length of string diverges like $1 / \varepsilon[10]$. From this we see that as $\varepsilon$ decreases, the string appears to fill space more and more densely.

A similar calculation can be performed for the longitudinal size of the string, and one finds that 11]

$\left\langle 0\left|\left(X^{-}(0)-x^{-}\right)^{2}\right| 0\right\rangle \sim \frac{1}{\varepsilon}$.

This shows that the usual Lorentz contraction along the direction of motion is eventually halted in string theory, and the string begins to grow like $1 / \varepsilon$.

Now let us return to the case of an external observer watching a string fall toward the event horizon of a large black hole. From Eq. 26, we saw that an external observer has a time resolution that decreases like $e^{-t / 4 G M}$. Thus, an external observer will see the mean squared radius of the string grow like $t / 4 G M$, while its total length and longitudinal spread will increase like $e^{t / 4 G M}$.

Using this, we see that the time to spread over the entire horizon is of order $t_{\text {spread }}=G^{3} M^{3}$, which is much less than the evaporation time $t_{\text {evap }}=G^{2} M^{3}$. In addition, the longitudinal spread causes the string to fill up a region near the horizon, where it appears to float.

The previous analysis shows that someone observing an infalling string will find that it appears to grow and cover the horizon, forming a sort of stringy goo just above the horizon [12]. One 
may rightly ask if there is any sense in which the information contained in the string state also is spread out, or thermalized. In ordinary field theory, degrees of freedom are independent of each other unless they have a non-vanishing commutator. It is then natural to ask, what is the analogous statement in string theory?

In work by Lowe and the authors [13], this question is addressed using light front bosonic string theory. The commutator of two component fields at equal light front time is shown to be non-vanishing to first order in the string coupling even when the arguments of the fields are spacelike (transversely) separated. This shows that the information in the string state also spreads.

In more recent work by Lowe, Polchinski, Thorlacius, and the authors [14], the commutator of appropriately dressed low energy component fields is calculated and shown to be nonzero when the fields are at different light front times. More precisely, suppose observer 1 at position $x_{1}$ stays in front of the horizon at some finite distance $\rho$, while observer 2 freely falls through the horizon. Let $x_{2}$ lie on the world line of observer 2 behind the horizon. Let $\phi\left(x_{1}\right)$ and $\phi\left(x_{2}\right)$ be component fields which are low energy in the frame of the respective observers. Then the matrix element

$$
\left\langle 0\left|\left[\phi\left(x_{1}\right), \phi\left(x_{2}\right)\right]\left[\phi\left(x_{2}\right), \phi\left(x_{1}\right)\right]\right| 0\right\rangle
$$

is shown to be nonzero to first order in the string coupling, and in fact grows like $e^{t / 4 G M}$. This growth can ultimately be traced to the Regge behavior of string scattering amplitudes, and the existence of the graviton in string theory.

The calculation of the size of a string in its ground state was performed using free string theory. We saw that because the length of string grows faster than the area it occupies, the string becomes dense as $\varepsilon$ decreases. When the transverse density becomes of order $1 / g^{2}$, interactions will become important, and our perturbative calculation can no longer be trusted. Presumably, at this stage non-perturbative string physics takes over and the density does not grow past the Planck density. We will have more to say about this later.

\section{Black Hole Entropy in String Theory}

It was stated earlier that black holes have an entropy, given by the Bekenstein-Hawking formula,

$S=\frac{A}{4 G \hbar}$,

where $A$ is the area of the horizon and we have made the factor of $\hbar$ explicit. It should be pointed out here that the fact that black holes have a nonvanishing entropy is not due to quantum mechanics. The fact that the entropy is finite is due to quantum mechanics, as can be seen in Eq. (36). As $\hbar \rightarrow 0$, the entropy diverges.

The fact that black holes have entropy has been an extremely confusing one, for at least two reasons. We ordinarily think of entropy as having to do with counting the number of degrees of freedom of a system, and since the entropy is proportional to the area of the horizon, it certainly seems as if we are counting the states of something near the horizon. Precisely what states are being enumerated, however, remains unclear. What makes this even more confusing is that we are attributing an entropy to something which is essentially a classical solution of the gravitational field equations, something which seems very much like a soliton, and we certainly do not ordinarily assign an entropy to a soliton.

On the other hand, it is possible to calculate the entropy of a field propagating on a fixed black hole background. We saw in Chapter 3 that the correct way to think of a quantum field propagating outside a black hole is as a thermal system, so we could follow 't Hooft and propose that the entropy of the black hole is nothing but the entropy of the thermal atmosphere of particles around the black hole. The trouble is, explicit calculation of the entropy of a massless, real free scalar field $\phi$ in the thermal or Unruh state outside a black hole shows that the entropy is quadratically divergent, and is given by

$S_{\phi}=\frac{c A}{4 \varepsilon^{2}}$

where $\varepsilon$ is a proper distance cutoff of the theory and $c$ is a numerical factor which depends on the 
precise form of the cutoff. This is in sharp distinction to Eq. (36), which is manifestly finite.

This raises the question, should we include this divergent entropy as part of the entropy of the black hole? If not, why not? The point is that the divergent entropy of the field $\phi$ is due to the enormous number of states that are available to the field near the horizon. This can be seen by explicit computation, but it is connected with the ideas introduced earlier of what happens to matter as it approaches the horizon. If particles are allowed to Lorentz contract to arbitarily small longitudinal extent, then an arbitrarily large number of them can be packed close to the horizon, giving a divergent entropy. These states are of extremely short wavelength, but do not carry a lot of energy because of the redshift phenomenon. The above paradoxes have led some physicists to abandon the connection between entropy and state counting, but we shall assume that it continues to hold for black holes, and see where that leads us.

Let us now consider how to calculate the entropy of a Rindler horizon. We have seen that one of the advantages of Rindler horizons over real black hole horizons is that we can use flat space, where we know basically how to perform calculations. The Rindler horizon is infinite in area, but we expect the entropy per unit area to be well defined.

One way of doing statistical mechanics for a canonical ensemble is to perform a Wick rotation to imaginary time, and then make the time variable periodic with period $\beta=1 / T$, where $T$ is the temperature of the canonical ensemble. Thus we will be dealing with Euclidean Rindler space, which has the line element

$d s^{2}=\rho^{2} d \theta^{2}+d \rho^{2}+d \vec{x}^{2}$,

where $\theta \in(0, \beta)$ is the Euclidean time variable and $\vec{x}$ denotes the transverse coordinates. The horizon has now been compressed to the surface $\rho=0$. If $\beta=2 \pi$, then Eq. (38) is nothing more than the line element for flat Euclidean space in cylindrical polar coordinates. For $\beta \neq 2 \pi$, the space has the geometry of the cross product of a cone with $D-2$ dimensional flat space. This geometry has a curvature singularity at the hori- zon. Since such a singularity was not present in the original spacetime, we must choose $\beta=2 \pi$, rederiving the Rindler temperature.

The Rindler Hamiltonian now generates rotations of the space, and the Unruh density operator is simply equal to

$\rho_{\text {Unruh }}=\frac{\exp \left(-\beta H_{R}\right)}{Z(\beta)}$,

where $Z(\beta)$ is the partition function,

$Z(\beta)=\operatorname{tr}\left(\exp \left(-\beta H_{R}\right)\right)$.

We now want to calculate the entropy for this density operator with $\beta=2 \pi$.

One way of calculating the entropy is to recall the thermodynamic relations

$$
\begin{aligned}
Z(\beta) & =\exp (-\beta F(\beta)) \\
\text { and } & \\
S(\beta) & =\beta^{2} \frac{\partial F}{\partial \beta} .
\end{aligned}
$$

In order to use these identities, however, we have to be able to vary $\beta$ away from $2 \pi$, which means we must do physics, either field theory or string theory, on a cone.

Let us imagine representing the partition function $Z(\beta)$ as a functional integral over all geometries with certain boundary conditions. The leading order contribution to the functional integral is simply $\exp \left(-I_{C}(\beta)\right)$, where $I_{C}$ is the Euclidean action evaluated for the solution of the classical equations of motion. (We are ignoring a possible cosmological constant term, which cannot contribute to the entropy anyway.) Thus we can write, to leading order, $\beta F=I_{C}$. The classical action is (ignoring surface terms, which do not play a role here)

$$
\begin{aligned}
I_{C} & =\frac{1}{16 \pi G_{0}} \int d^{4} x \sqrt{g} R \\
& =\frac{(2 \pi-\beta) A}{8 \pi G_{0}},
\end{aligned}
$$

where $A$ is the area of the horizon and $G_{0}$ is the bare gravitational coupling constant. This then gives an entropy

$S_{0}=\frac{A}{4 G_{0}}$. 
It cannot be emphasized enough that we do not have a statistical interpretation for the entropy here. There is no mention of what states are being counted. This is simply a formal procedure-we get the correct answer, but we don't really know what it means 15.

If we add a free scalar field $\phi$ to the system, the entropy of the field can be calculated in the same way. This was first done by 't Hooft [16], and later by other authors. The action can be represented as a sum of first-quantized particle paths, so we can identify each contribution with a set of paths. The set of paths which do not encircle or touch the horizon do not contribute to the entropy. To see why, consider a path in this set. The action of the (local) path cannot depend on the (global) value of $\beta$ unless the path is somehow entangled with the surface $\rho=0$. Integrating the center of mass of a specific configuration around the spacetime gives a volume factor proportional to $\beta$, which is then divided out to obtain the free energy, and the result is thus independent of $\beta$, and so contributes zero to the entropy. On the other hand, paths which do encircle or touch the origin can contribute to the entropy, and these are the states which are being counted. The calculation is easily done, and Eq. (37) is recovered.

If we add this new contribution to the entropy, we would apparently violate the universality of the Bekenstein-Hawking entropy. But we can write the sum in a suggestive form [17,

$S_{0}+S_{\phi}=\frac{A}{4}\left(\frac{1}{G_{0}}+\frac{c}{\varepsilon^{2}}\right)$,

and remember that the bare coupling constant is renormalized. We can argue that what is going on is in fact the renormalization of the coupling constant by calculating the renormalization of $G_{0}$ for an arbitrary geometry and showing that the coefficients are the same, and explicit calculation in perturbation theory shows that this is in fact the case 18, 19. So what we have is essentially a low energy theorem, stating that it is the fully renormalized gravitational coupling which enters the Bekenstein-Hawking formula. In fact, since we do not yet actually have a quantum theory of gravity, the above can be viewed as a sort of consistency condition on the theory. The main result we should take away from this is that the question of finiteness of the black hole entropy is intimately entanglement with the ultraviolet behavior of the theory of quantum gravity, and is not something that can be understood using only low energy effective field theory.

Conspicuous by their omission from the previous discussion are graviton loops. These loops are very complicated, and in fact lead to disasters. These disasters are associated with infrared divergences, which are the manifestation of the Jeans instability. The nature of these divergences can be understood by making an analogy to a plasma. The Boulware vacuum is the vacuum defined by eliminating all of the thermal particles from the Unruh vacuum-it is essentially the Rindler Fock space vacuum. This vacuum must have a large and negative Minkowski energy, because the Minkowski vacuum $|0\rangle$, which corresponds to the Rindler space Unruh vacuum, has no Minkowski energy. We must therefore attribute to truly empty Rindler space a large and negative energy density. Starting with the Boulware vacuum, we can fill it up with thermal particles until reaches the Unruh state, and the energy density vanishes. This is somewhat analogous to having a system with a uniform positive charge density, and filling up the system with electrons until the net charge is zero. People who study the statistical mechanics of plasmas know that you have to be careful in dealing with the Coulomb force, and one must regulate the infrared tails in order to define the statistical ensemble. For example, varying the charge density away from its background value will create infrared divergences unless the Coulomb force is cut off.

This analogy suggests the following order of operations in calculating the entropy of the horizon. We must first infrared-regulate the theory, by cutting off the long range gravitational field. Having done this, we can vary $\beta$ away from $2 \pi$ to calculate the entropy. After obtaining the functional form of the entropy, we evaluate it for $\beta=2 \pi$. Finally, we take away the regulator, and hope that the answer remains well defined.

The story is slightly more complicated for string theory [17]. As we saw earlier, for $\beta \neq 2 \pi$, the geometry is the product of a cone with flat 
space, and this background geometry does not satisfy the conditions for conformal invariance of the world sheet theory, and the world sheet theory is not ultraviolet finite. To define the theory offshell, we must introduce a world sheet ultraviolet regulator into the theory. But as we saw before, this has exactly the effect of cutting off the spacetime theory in the infrared! Thus, by introducing the world sheet regulator we simultaneously define the theory off-shell and eliminate the Jeans instability, so we can expect that the statistical ensemble will be well defined. Of course, this is a conjecture about how to define string theory off shell, so we should check that our answers are independent of this prescription.

So our task now is to calculate the genus expansion of the free energy of strings in a conical background, which will take the form

$\beta F(\beta)=\sum_{n=0}^{\infty} g^{2(n-1)} Z^{(n)}(\beta)$

where $g$ is the string coupling constant and $Z^{(n)}$ is the partition function for the two dimensional theory on a world sheet of genus $n$. Consider first the genus zero term. If we imagine using a lattice regulator, so that the world sheet is composed of $N$ points, then the partition function of the world sheet theory reduces to a product of ordinary coupled Gaussian integrals. Because of the exponential fall off of the integrands, there can be no nonlocal behavior introduced into the integrals, so on general grounds, we can expand the partition function in local geometric invariants as

$Z^{(0)}=-\frac{1}{16 \pi G_{0}}\left[\int d^{4} x \sqrt{g} R+Q\right]$,

where $Q$ contains all the other terms which enter the expansion. (We are again dropping any cosmological constant term, which cannot contribute to the entropy.) In general, $Q$ will depend in a singular way on the world sheet regulator and on the conformal degrees of freedom, but it is important to note that the first term does not depend on either. These singular terms can be interpreted as the residue of the graviton, whose long range field has been truncated by the regulator.

The contribution from the first term of Eq. (46) is precisely the term we found before, and gives an entropy equal to Eq. (43). For the remaining terms, it is easy to argue that they either vanish by integration by parts, or are proportional to $(2 \pi-\beta)^{2}$, and so will vanish when we set $\beta=2 \pi$. Thus, the only term which can contribute to the entropy is the first term, which gives Eq. (43), and we can safely remove the world sheet regulator. So the genus zero contribution to the entropy of the horizon is precisely the BekensteinHawking entropy, with the bare coupling constant $G_{0}$. The analysis goes through essentially unchanged for the higher genus contributions, although for higher genus we must also regulate the corners of the moduli space.

Since the scattering amplitudes of string theory are finite, we are guaranteed that when we sum up the contributions, the horizon entropy per unit area is finite order by order in perturbation theory, so string theory avoids the problem of any infinite contributions to the entropy.

The answer we obtained for the contribution to the horizon entropy from the genus zero partition is exactly the same as that obtained from the classical action of the gravitational field earlier. In the latter case, however, we saw that there was no way to identify which states the entropy was counting. Let us now see if we can discover what states we are counting in string theory.

As with the field theory case, the only string graphs which can contribute to the entropy are those which are somehow entangled with the horizon. An example for genus zero is shown in Fig. (1. In order to determine the state which this graph corresponds to, we simply take a slice of constant Euclidean time $\theta$. It is easy to see that the state is described by an external observer as an open string with both ends frozen to the horizon. This state could equally well be described as a closed string lying partially behind the horizon. So the entropy corresponds to the states of fluctuations of open strings pinned to the horizon surface.

Taking the usual field theory limit $\alpha^{\prime} \rightarrow 0$, these configurations degenerate to points on the horizon, and their interpretation as states is lost. In this case, we simply have to say that there is an entropy whose origin cannot be understood from the low energy theory. 


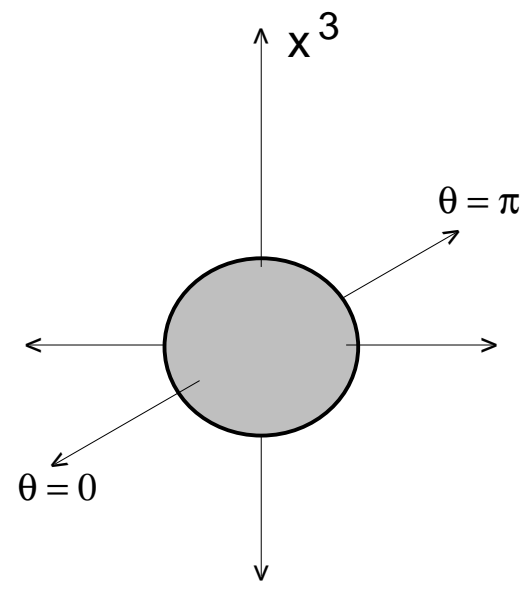

Figure 4. A genus zero string graph which contributes to the entropy.

Fig. 5 and Fig. 6 show genus one diagrams which contribute to the entropy. Fig. 5 shows a diagram whose state-counting interpretation is simply a closed string which remains outside the black hole. The $\alpha^{\prime} \rightarrow 0$ limit of this diagram simply corresponds to a particle which remains forever outside the black hole. Fig. 6, however, cannot be identified as a single state, but only as an interaction between a string frozen to the horizon and a string outside the black hole. This term should not be thought of as the entropy of anything, but as a correction term. In the $\alpha^{\prime} \rightarrow 0$ limit, these diagrams must be associated with contact terms with the horizon. These terms have been discovered in ordinary quantum field theory 19.

Thus we see that the stranded strings can interact with each other and with strings outside the black hole, so what we have is a complicated many body problem near the horizon with a large number of degrees of freedom. This system, as with any other complicated system, is capable of storing, thermalizing, and re-emitting information which it comes into contact with.
Figure 5. A genus one string graph showing a string which remains permanently outside the horizon.

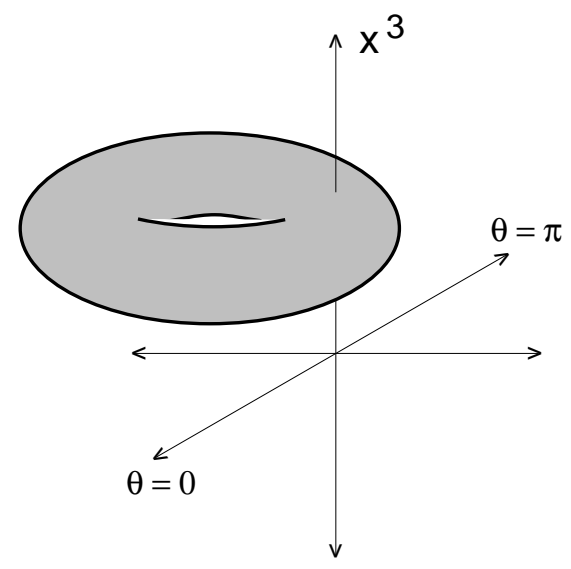

Figure 6. A genus one string graph showing an interaction between a stranded string and an external string. 


\section{The Holographic World}

In the previous chapters, we have seen that strings exhibit a variety of new and interesting phenomena which are relevant to the physics of black holes. These include the observed transverse spreading, the reversal of longitudinal Lorentz contraction, the fact that string field commutators do not vanish at spacelike separation, the finiteness and interpretability of horizon entropy in string theory. The task at hand now is to understand what all these phenomena imply for a non-perturbative formulation of quantum gravity.

To begin, let us count the number of degrees of freedom of string theory. To do so, we need to make sure that any gauge symmetries of the theory are completely fixed, since gauge symmetries are not really symmetries at all, but redundancies in the description of the theory. The only such complete gauge fixing of string theory that we know of is the light front gauge.

As we have seen, in light front gauge the longitudinal momentum of the string is uniformly distributed over $\sigma$, and the conservation of longitudinal momentum is equivalent to the conservation of the total parameter length. Let us regulate the string theory by dividing the string into $N$ pieces, each with longitudinal momentum $\varepsilon=p^{+} / N$. It is then true that in any string interaction, the number of bits of string, or partons, is conserved.

The free string Hamiltonian in light front gauge is then given by

$H_{0}=\frac{1}{2 \varepsilon} \sum_{i}\left[\vec{P}(i)^{2}+(\vec{X}(i+1)-\vec{X}(i))^{2}\right]$.

The interaction Hamiltonian will include terms which generate the usual splitting and joining of strings, as well as contact terms which are necessary for gauge invariance.

There are some interesting aspects of the regulated light front theory. First of all, the full Hamiltonian depends only on the transverse directions. The interaction Hamiltonian contains only delta-functions of transverse position. There is no part of the Hamiltonian which depends on the longitudinal direction. On the face of it, this fact seems very odd, and seems to indicate a se- vere degree of non-locality in the theory. We will soon see the significance of this.

Second, if one adds more partons (increasing $N$ and simultaneously decreasing the minimum longitudinal momentum $\varepsilon$ ) one finds that the string does not develop short distance structure in spacetime. Instead, one obtains lots of very large loops, all with radius of curvature of order $\sqrt{\alpha^{\prime}}$ [10]. These loops are contained within an area of order $\log (N)$. Thus, as we increase the resolution, we do not obtain lots of small wiggles around a nice, limiting curve, but instead find a wildly undulating mess of string which grows very dense.

Finally, independent of string theory, suppose that the number of degrees of freedom $N$ necessary to describe Planck scale physics inside a volume $V$ was proportional to the volume. This leads immediately to a contradiction with black hole physics and the Bekenstein-Hawking entropy [20]. For suppose we have some mass $M$ inside $V$. If the boundary of $V$ has area $A$, drop in a shall of mass that causes a black hole of area $A$ to form. Then the Bekenstein-Hawking entropy formula tells us that the number of degrees of freedom necessary to describe Planck scale physics in the volume after we have added some mass is proportional to $A$. Thus the entropy has decreased, in direct contradiction to the second law of thermodynamics. Thus the maximum number of non-redundant degrees of freedom necessary to describe Planck scale physics inside a volume $V$ must in fact be proportional to the area of the boundary.

Moreover, this suggests that, given a system inside a volume, there exists a mapping of the system onto a set of surface degrees of freedom which preserves the information contained within the original system. In particular, if we place a large screen outside a black hole, there is a mapping from each Planck area on the event horizon to a Planck area on the screen.

Are these ideas crazy? It turns out that by using the focussing theorem of general relativity, it is easy to show that, at least at the classical level, one can in fact construct an injective map from the surface of a black hole to the surface of a flat screen far away. Similarly, one can show 
that no matter how many black holes one puts in a row, there always exists an injective map from the union of the event horizons to the screen. The conclusion, then, is that gravity should be able to be formulated as a $2+1$ dimensional theory with one degree of freedom per Planck area [20].

The significance of the strange non-locality we encountered in the light front formulation of string theory is now becoming more clear. As we saw above, the light front formulation of string theory, which is fully gauge fixed and contains no redundant degrees of freedom, does not depend at all on the longitudinal direction - it is a $2+1$ dimensional theory. Thus string theory is in some sense already a holographic theory.

We saw earlier that as resolution time decreases (energy increases) the string becomes more and more dense in spacetime. When the transverse density $\rho$ becomes of order $1 / g^{2}$, interactions become important, and we expect non-perturbative effects to enter. This is, in fact, how the Planck scale enters string theory. In order for string theory to be consistent with the idea that there is no more than one degree of freedom per Planck area, the non-perturbative effects must be such that the string bits become repulsive. We can guess that the mean squared radius must start to obey $R^{2} \sim N$ beyond a certain momentum, presumably of order the Planck energy. This implies that we should expect a cross section for Planckscale scattering which grows like the energy at high energy.

In conclusion, we see that string theory, if formulated as a $3+1$-dimensional theory, must have so much gauge freedom that a complete gauge fixing will reduce the theory to a discrete $2+1$ dimensional theory. This amount of gauge symmetry is far larger than anything we have yet encountered. We can easily conjecture that the myriad dualities being discovered may be providing a first glimpse at how this gauge symmetry is implemented in string theory.

\section{REFERENCES}

1. J. J. Atick and E. Witten, Nucl. Phys. B310 (1988) 291.

2. See, e.g., D. J. Gross and P. F. Mende, Nucl.
Phys. B303 (1988) 407.

3. S. W. Hawking, Comm. Math. Phys. 43 (1975) 199.

4. L. Susskind and L. Thorlacius, Phys. Rev. D49 (1994) 966.

5. K. S. Thorne, R. H. Price, and D. A. MacDonald, Black Holes: The Membrane Paradigm, Yale University Press, 1986.

6. J. D. Beckenstein, Phys. Rev. D7 (1973) 2333; Phys. Rev. D9 (1974) 3292.

7. S. W. Hawking, Phys. Rev. D13 (1976) 191.

8. L. Susskind, L. Thorlacius, and J. Uglum, Phys. Rev. D48 (1993) 3743.

9. D. N. Page, Phys. Rev. Lett. 71 (1993) 1291.

10. M. Karliner, I. Klebanov, and L. Susskind, Int. Jour. Mod. Phys. A3 (1988) 1981.

11. L. Susskind, Phys. Rev. D49 (1994) 6606.

12. A. Mezhlumian, A. Peet, and L. Thorlacius, Phys. Rev. D50 (1994) 2725.

13. D. A. Lowe, L. Susskind, and J. Uglum, Phys. Lett. B327 (1994) 226.

14. D. A. Lowe, J. Polchinski, L. Susskind, L. Thorlacius, and J. Uglum, Black Hole Complementarity vs. Locality, preprint NSFITP-95-47, UCSBTH-95-12, SU-ITP-95-13, hep-th/9506138.

15. L. Susskind, Some Speculations About Black Hole Entropy in String Theory, Rutgers University preprint RU-93-44, August 1993, hepth/9309145.

16. G. 't Hooft, Nucl. Phys. B256 (1985) 727.

17. L. Susskind and J. Uglum, Phys. Rev. D50 (1994) 2700.

18. J.-G. Demers, R. Lafrance, and R. C. Myers, Phys. Rev. D52 (1995) 2245;

D. Kabat, Nucl. Phys. B453 (1995) 281.

19. D. Kabat, S. Shenker, and M. J. Strassler, Black Hole Entropy in the $O(N)$ Model, Rutgers University preprint RU-95-34, hepth/9506182.

20. L. Susskind, J. Math. Phys. 36 (1995) 6377. 